\title{
SECOND GENERATION MOSAIC:A NOVEL MECHANISM BASED ON REFERENCE DATABASE FOR MAP UPDATING
}

\author{
HuGaoxiang* ${ }^{* a}$, Frank Bignone ${ }^{\mathrm{b}}$
}

${ }^{\mathrm{a}}$ EADS-CHINA; ${ }^{\mathrm{b}}$ Astrium geo-information services

KEYWORDS: Map Updating, Mosaic, Parallel Processing, Orthoimage Mosaic

\begin{abstract}
:
A totally new automatic workflow mechanism, named Second generation Mosaic module, based on the well-known Pixel Factory system, will be introduced here, which enables existing digital orthoimages and mosaics to be quickly updated. The process extracts all required parameters from the reference database to be able to perform automatic bundle adjustment and radiometric adaptation.In this paper, two examples for both satellite data and aerial data processed based on this mechanism will be presented here and discussed. Finally, the cost reduction will also be analysed according to the real mapping updating project.In a final statement, this paper will present an integrated solution named second generation mosaic module based on the well-known Pixel Factory system which is completely dedicated to fast processing of photogrammetric products Thanks to this integrated hardware and software solution, it is even possible to manage large data volume quickly in order to have precise map updating information as soon as possible after acquisition.
\end{abstract}

\section{SECOND GENERATION MOSAIC CONCEPT AND PRINCIPLE}

SGM (Second Generation Mosaic) is a global process that allows generating rectified imagery automatically by using available referenced imagery as a source for georeferencing, rectifying and dodging the new imagery. The principle is to use the referenced imagery to create a pattern of quasi-GCPs that will be matched between the new imagery and the referenced imagery. This should allow adjusting the new imagery without any additional GCPs. It combines three different tools in a single package:

- $\quad$ A database management tool;

- $\quad$ A workflow management tool;

- Integrated activities that allow the automatic processing of data: automatic bundle adjustment, Bundle Quality Check.

The database management tool allows to maintain a repository
The database management tool allows to maintain a repository of imagery (ground orthos and elevation images), GCPs and to manage queries. Imagery will be extracted (and filtered) according to the project area and user specifications. Once the new imagery is imported, the Automatic Bundle Adjustment activity will:

- $\quad$ create and match tie points between the new images;

- $\quad$ create and match tie points between the new images and the georeferenced image (or use existing GCPs); adjust the block and filter again tie points using user defined specifications.

The $\mathrm{Bu}^{1}$ ndle Quality Check activity is a stop/go point in the workflow. When the residuals exceed a user defined threshold, the process will halt and the user should take corrective action. If the residuals are under the threshold, the Validate Bundle Adjustment activity will be executed and the rectification sequence will start. ${ }^{[1][2]}$. 


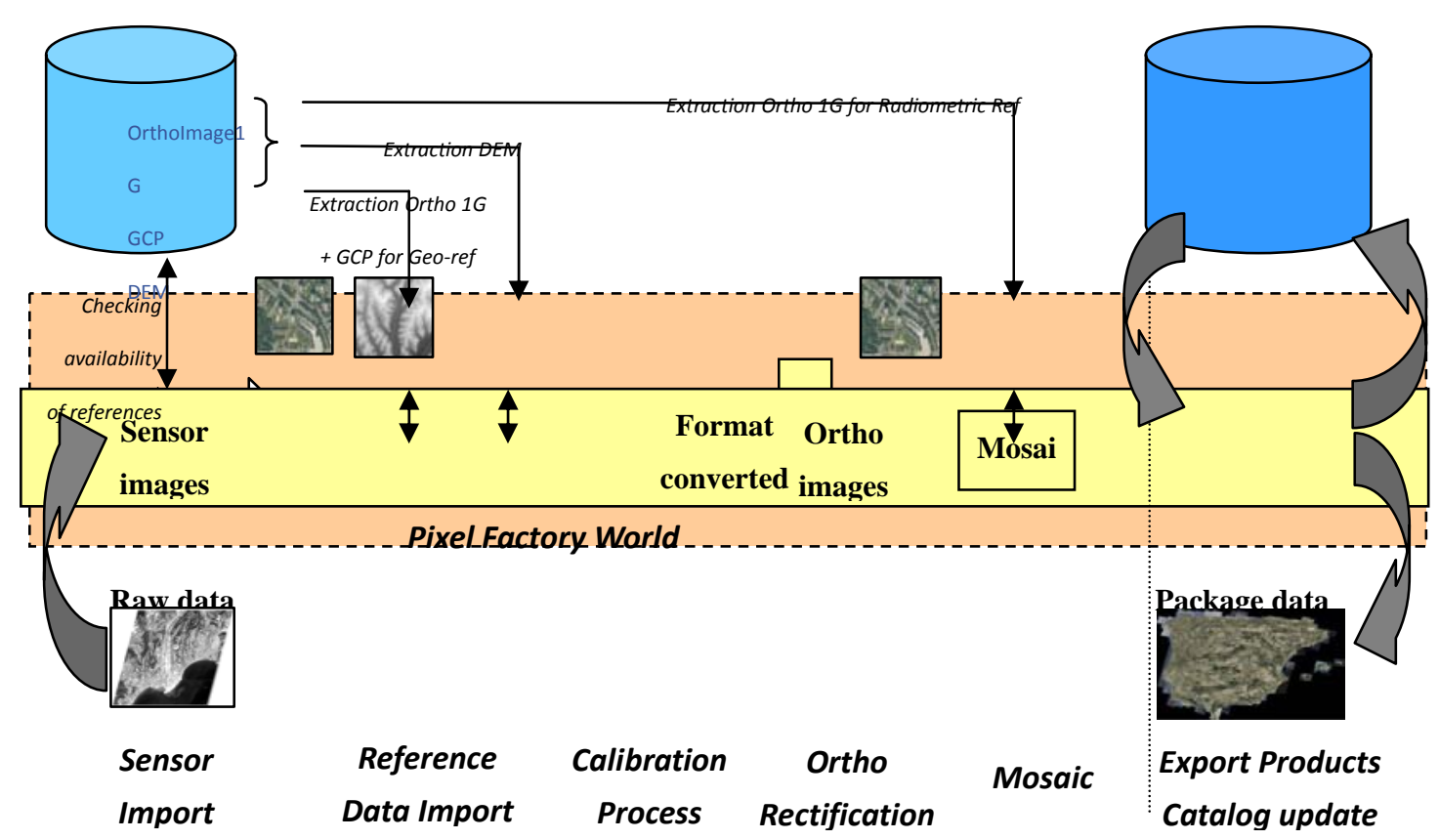

Figure 1. Second Mosaic Generation (SGM) principle

\section{REFERENCE DATABASE CHARACTERISTICS}

There is no need to convert the project data to the geographic WGS84 coordinates systems, or to actually run a project in that system either. This footprint is an additional tag that is used to speed up the identification of relevant data. The reference database used by SGM is a repository of three types of data:

- $\quad$ ortho images

- GCP

- $\quad$ elevation images (DEM)

There are very few restrictions about the data itself. All cartographic systems are virtually supported. Elevation data should be in raster format. The reference database stores the characteristics of the data and a link to each image. Therefore, the imagery must be stored on an accessible file system. In order to extract relevant data, the footprint of each data is also stored in geographic / WGS84 coordinates.

Extracting data from the database is done by using the Get Reference from a reference database. This activity will:

- $\quad$ check that there is data available;

- filter the data according to various user defined criteria (such as the source of data, its accuracy etc.);
- check that the resulting set of data covers the requested $\mathrm{AOI}$;

- extract the data, resample and convert it into the project coordinate system.

While it is possible to have different ortho images overlapping each other and use all of them for the bundle adjustment, elevation data will be merged and resampled to form a single elevation image.

\section{GENERATING GCPS}

Ground control point might be of two types: quasi-GCP and true GCP. In SGM, a GCP comprises a set of coordinates, an accuracy value and an image tile. The tile is extracted from the ortho image used as reference. When measuring or adjusting, no distinction is made between the points. The only difference might be the accuracy value attached to the coordinates.

Quasi-GCP are points that are created according to a user defined pattern. These tie points are matched between the reference images and the new imagery. The planimetric coordinates are interpolated in the reference ortho image, and 
the elevation is interpolated from the DEM. After the matching, the accuracy attached to the coordinate will be the accuracy of the reference data.

True GCP are points that have been previously measured in the field, or have been selected by an operator from a reliable data source (e.g. high resolution ortho image, map etc.). An image tile from a reference image must be manually attached to those points beforehand, so that they can be matched accurately on the new image.

\section{AUTOMATIC WORKFLOW ENGINE}

This workflow allows creating the customized workflow, and all the photogrammetric process activities can be interconnected using the graphical easy to use workflow editor. It can help fully automatic photogrammetric process, and even a push mode embed the whole module will help the satellite receiving station for automatic product updating.

The workflow comprises three groups of activities:

- Preparation: importing new imagery; extracting data from the database;

- Bundle adjustment: setting up the project, creating tie points, optimizing parameters, validating the results

- Rectifying the new imagery: creating a DSM/DTM if necessary, creating ortho images, mosaicking and balancing. In most cases, you might use an existing or predefined workflow and assign new settings. Otherwise it is suggested that you duplicate and modify an existing one

A workflow consists in launching automatically pre-defined tasks. The workflow engine accepts:

- $\quad$ Parameter binding

- $\quad$ Parallels steps processing

- Conditionals branches for correction

\section{USE CASE}

In this paper, two examples for both satellite data and aerial data processed based on this mechanism will be presented here and discussed. Finally, the cost reduction will also be analysed according to the real mapping updating project.

\subsection{Aerial dataset usecase}

\subsubsection{Dataset: The provided dataset consists of:}

90 Level3 RGB UCD images (around $80 \%$ and $80 \%$ overlap between images).

Exterior orientation data in orima format (from other software).

Camera Calibration data.

Reference data from RefereceDB

DEM,DSM

TrueOrtho

Color Reference ortho images.

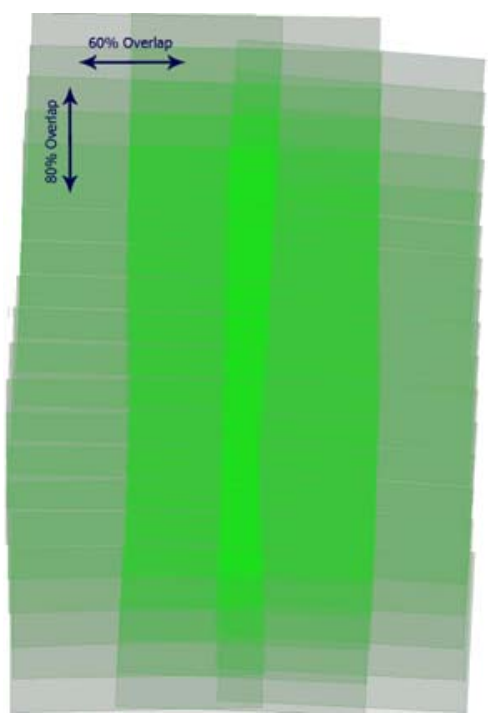

Figure 2. The outline of the provided data

5.1.2 Automatic workflow settings: Basically, in workflow definition editor, four steps are needed:

1. Create a new workflow definition or import an existed one.

2. Select the activities that could be used in your workflow definition

3. Draw the graphic workflow

4. Make links between related activities, andspecific necessary constraint.

Hereafter is the complete workflow set in workflow editor for this UCD processing. 


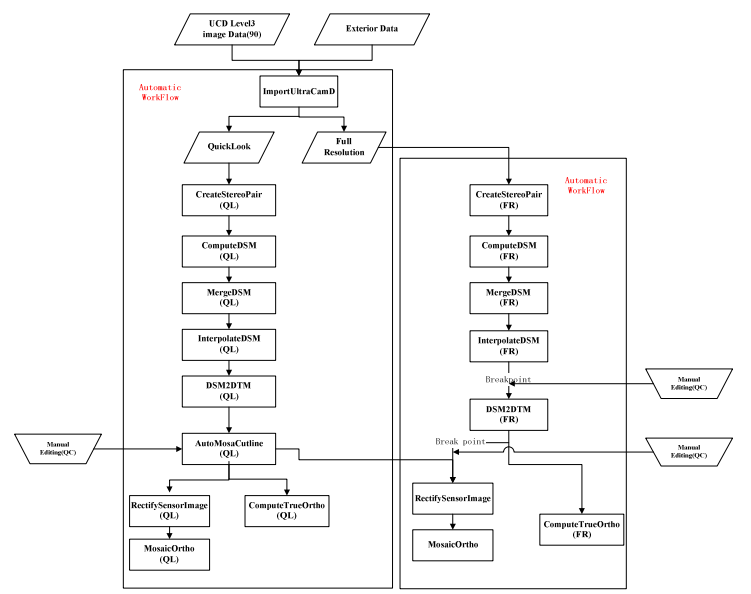

Figure 3. The footprint of UCD images

\subsubsection{Bundle Adjustment based on reference data: For the} bundle adjustment, as the reference ortho images and elevation data are extracted from database, all tie points are matched between the reference images and the new imagery. The planimetric coordinates are interpolated in the reference ortho image, and the elevation is interpolated from the DSM. After the matching, the accuracy attached to the coordinate will be the accuracy of the reference data. The bundle adjustment was then realized by using those automatic tie-points, and by optimizing the exterior orientation (X,Y,Z,omega,phi and kappa) for each image. ${ }^{[3]}$

Also, an automatic filtering technique was applied in order to remove blunders gradually until the accuracy can be achieved.

\begin{tabular}{|l|l|l|l|}
\hline \multicolumn{4}{|l|}{ Control points coordinates residuals(unit:meter) } \\
\hline XYZ bias & -0.00045 & 0.00009 & -0.001695 \\
\hline XYZ std & 0.08331 & 0.04779 & 0.091288 \\
\hline XYZ max & 0.16227 & 0.0669 & 0.150933 \\
\hline
\end{tabular}

\begin{tabular}{|l|l|l|}
\hline \multicolumn{3}{|l|}{ Xy residuals (unit: pixel) } \\
\hline xy bias & -0.000011 & 0.000008 \\
\hline xy $\quad$ std & 0.166236 & 0.179751 \\
\hline Xy $\quad$ max & 0.901190 & 0.944950 \\
\hline
\end{tabular}

Table 1. Accuracy of the UCD project

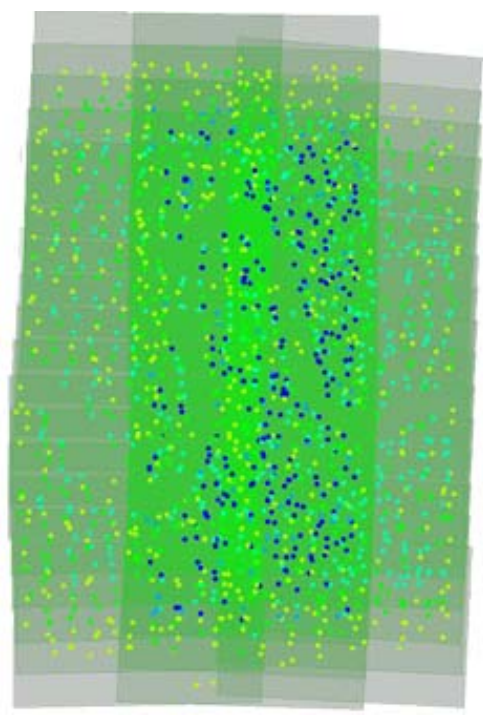

Figure 4. Tiepoints of UCD project

5.1.4 Digital Surface Model: The Digital Surface Model was computed by merging all individual Digital Surface Models computed from all reasonable stereoscopic pairs of input aerial images. After this fusion task, automatic filtering is applied in order to remove remaining blunders in the computed DSM.

The next figure displays the filtered DSM (no values are outlined in red) and the corresponding interpolated DSM (shaded display).

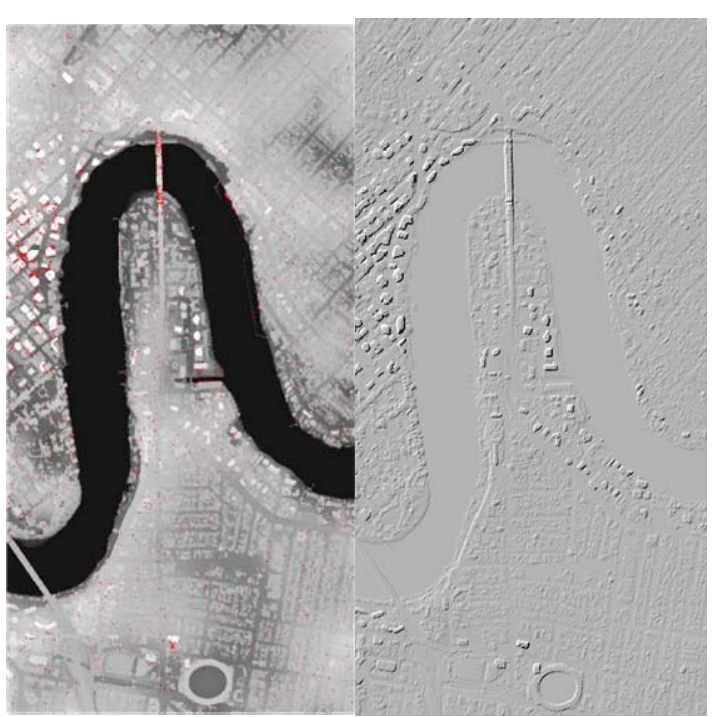

Figure 5. DSM automatically extracted from initial aerial images (in left image, red areas are point with no value) 
The quality of this DSM is correct, nevertheless height restitution inside the dense CBD (Center Business Distric) is impaired with the lack of overlap across track $(60 \%$ in the processed dataset instead of $80 \%$ ). The overlap along track is too much and $70 \%$ will have been enough.

5.1.5 GroundOrtho Mosaic: The ground orthoimage mosaic was processed by using:

- Initial RGB digital aerial images after bundle adjustment,

- DTM for image rectification,

- DSM for cut-line generation in urban areas.

- $\quad$ Reference ortho for color balance

The first step of the processing is to compute individual image rectification. Then based on those images, the DTM / DSM, SGM can automatically compute a cut-line that will optimize:

- Radiometric changes between neighbouring images,

- Angle of view in order to privilege most nadir point of view,

- Occlusion areas in order to avoid cut-lines crossing occluded areas in images.

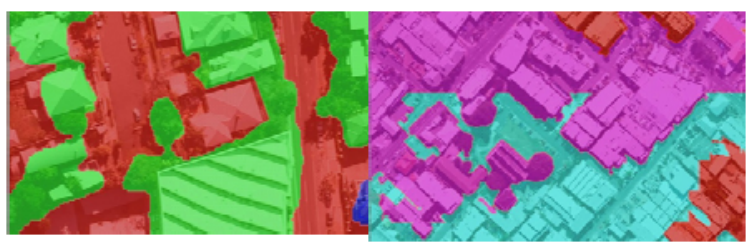

Figure 6. Mosaic with overlaid cut-line (each color is a different image)

After cut-line computation, the complete mosaic is computed with:

- Mosaic generation,

- Local radiometric equalization between images,

- Global radiometric equalization based on reference ortho images,

- $\quad$ Fuzzy margin along cut-lines.

All those steps are done in the same time, thus allowing faster computation. The radiometric equalization permits to optimize image radiometric difference at the same time globally based on the radiometric information (contrast and luminance) from reference ortho imagesand locally (dodging of image difference).

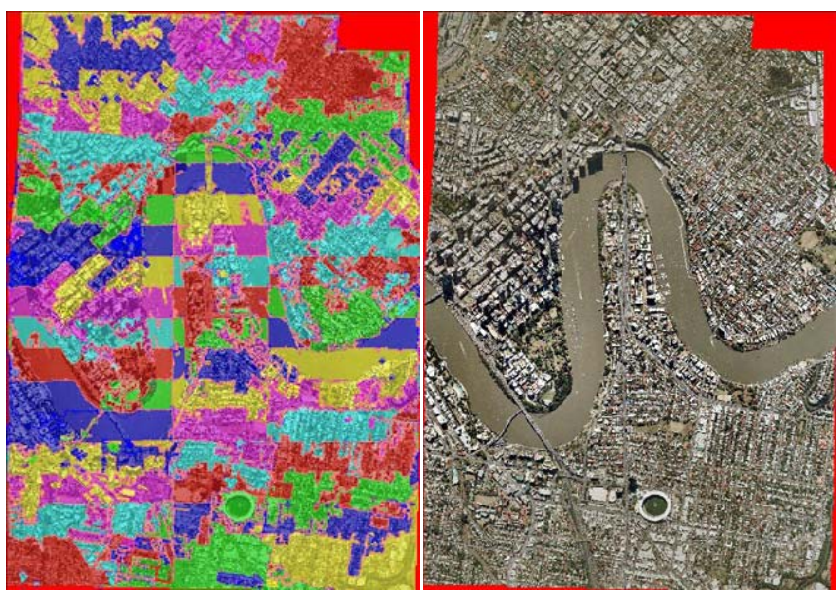

Figure 7. Computed GroundOrthoimage (left: cutline, right: mosaic)

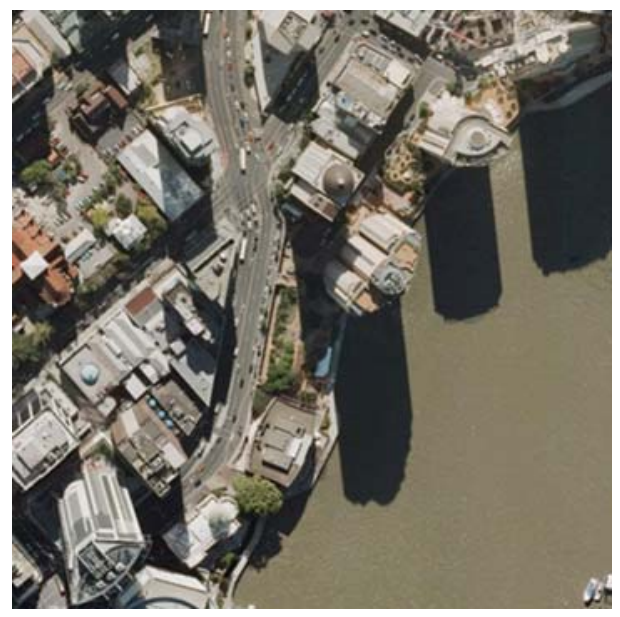

Figure 8. part of Mosaic

\subsection{Satellite data usecase}

SGM also has a potential use for map updating from satellite images as similar to the workflow as Aerial dataset. Amosaic processing based on mixed satellite dataset, 4 scenes SPOT5(XS1 XS2 XS3) and 2 scenes ASTER(vrni1 ) is taken. 

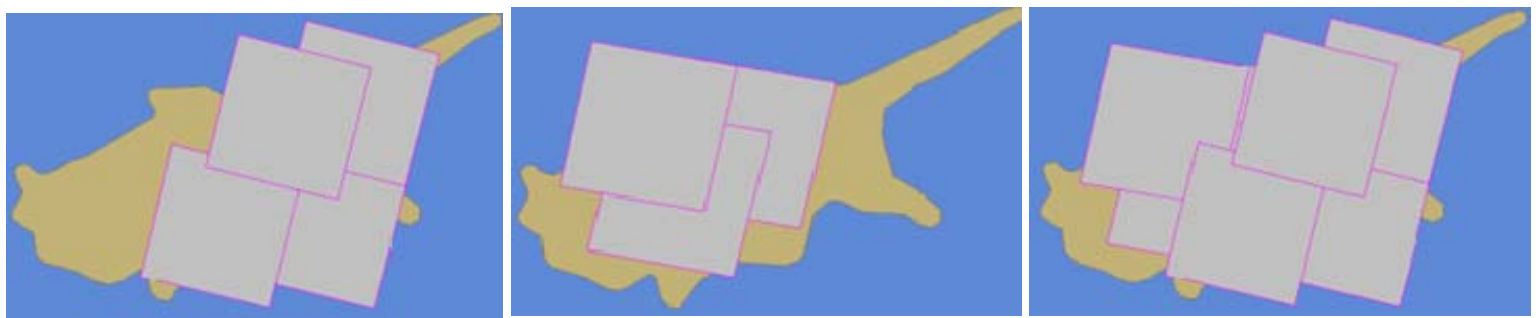

Figure 9. Inputdataset (Left:Spot5,Middle:ASTER,Left:Spot+ASTER)

The whole workflow desgined from data input to the final output is drawn below:

SPOT5+ASTER SGM Mosaic WorkFlow

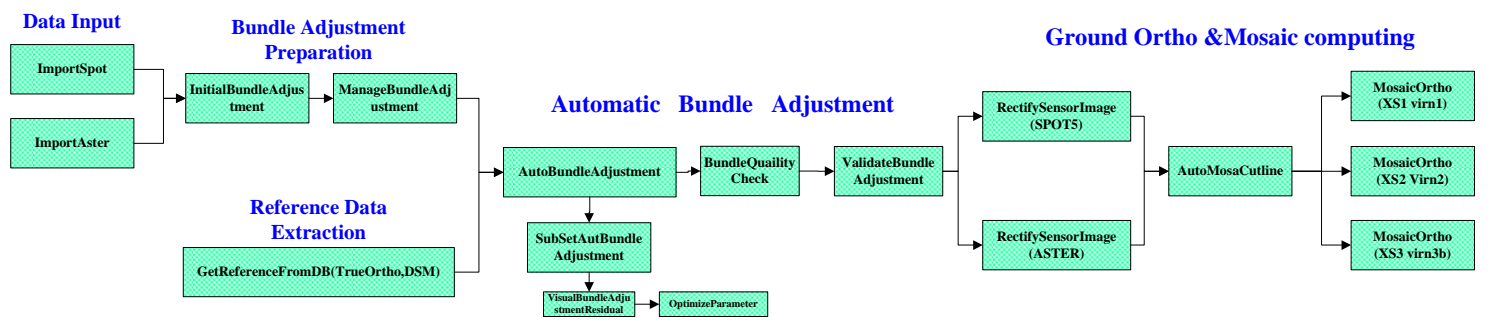

Figure 10. Workflow of the SPOT+ASTER SGM Mosaic

Here is the multi-sensor bundle adjustment result:

\begin{tabular}{|l|l|l|l|}
\hline \multicolumn{4}{|l|}{ Control points coordinates residuals(unit:meter) } \\
\hline XYZ bias & 0.000019 & -0.000213 & 0.145437 \\
\hline XYZ std & 0.077547 & 0.067593 & 3.444026 \\
\hline XYZ max & 0.418965 & 0.602366 & 9.117601 \\
\hline
\end{tabular}

\begin{tabular}{|l|l|l|}
\hline \multicolumn{3}{|c|}{ Image coordinates residuals (unit: pixel) } \\
\hline $\mathbf{x y} \quad$ bias & 0.000043 & 0.000004 \\
\hline $\mathrm{xy} \quad$ std & 0.146520 & 0.144642 \\
\hline $\mathbf{X y} \quad$ max & 0.683041 & 0.737197 \\
\hline
\end{tabular}

Table 2. Adjustment result of the UCD project

Thanks to the unique seamless automated image mosaicking techniques, though the color difference between ASTER and SPOT5,the finally mosaic still look quite homologous.
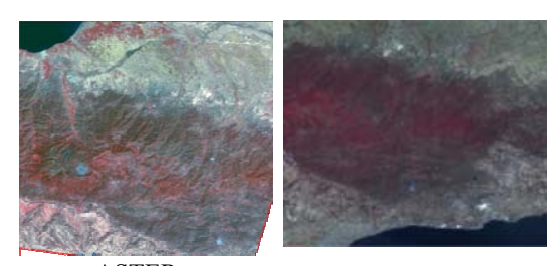

ASTER SPOT5(XS1 XS2 XS3)

(Vnir3n vnir2 vnir1)
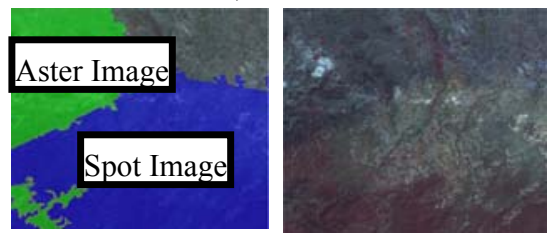

Mosaic Cutline

Mosaic

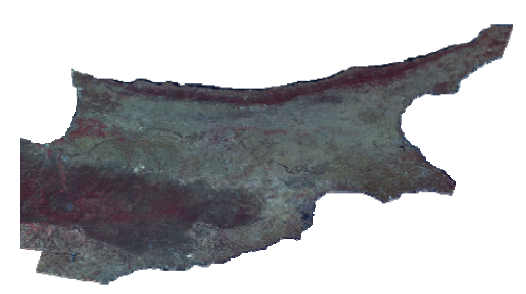

Mosaic Result

Figure 11. Finally mosaic result 


\section{CONCLUSION}

The proposed methodology provides an efficient way for any kinds of data/map updating. The Second Generation Mosaic (SGM) Add-on module is a cost-effective new module for Pixel Factorywhich enables existing digital orthoimages and mosaics to be quickly updated. The process extracts all required parameters from the reference database to be able to perform automatic bundle adjustment and radiometric adaptation. The time to produce a new mosaic is spectacularly reduced and a perfect fit between new and existing data is obtained. The Add-on can be used for both satellite and aerial imagery for every kind of production environment (including the Ground receiving station).And cost reduction in producing SGM imagery results from eliminating 4 main activities:

- Ground survey with GPS capture,

- Geometric/Aerial Triangulation,

- DEM acquisition and processing,

- Radiometric and atmospheric correction.

Cost reduction by drastically reducing sidelap and overlap of a flight if stereo pairs are not desired. Finally, it is between $20 \%$ and $80 \%$ less expensive than "first generation" mosaics. This new method of production addresses budget constraints and you preserve your original investment. Previously-completed mapping with all its features will be updated by the new second-generation imagery.

\section{REFERENCES}

[1] Manuel Jauregui, A procedure for map updating using digital momo-plotting and DTMs,IAPRS,Vol.32,Part 4"GIS-Between Vision and Applications",Stuttgart,1998

[2] Ma Li,Physical barrier detection for updating of navigation databases from high resolution satellite imagery,ISPRS Workshop on Updating Geo-spatial Databases with Imagery \& The 5th ISPRS Workshop on DMGISs

[3] Frank Bignone. Processing of stereo-scanner: from stereo plotter to Pixel Factory. Photogrammetric Week, Stuttgart, 2003 